separation between oestrous behaviour and vaginal and uterine state. Young et al. ${ }^{3}$, working with guinea-pigs, also found ovarian activity and oestrous behaviour to be imperfectly correlated, and, furthermore, their results show that the relationships between the three measures of ovarian activity were also variable. It is very probable that during the laying season of a bird a number of potential eggs are lost through lack of coordination between the ovary and oviduct.

D. G. M. Wood-Gush

A. B. GrLbert

Agricultural Research Council,

Poultry Research Centre,

West Mains Road, Edinburgh.

${ }^{1}$ Gilbert, A. B., and Wood-Gush, D. G. M., J. Reprod. Fertil., 5, 451 (1963).

${ }^{2}$ Michael, R. P., Science, 136, 322 (1962). 3 Young, W. C., Dempsey, E. W., Myers, H. I., and Hagquist, C. W., Amer. $J$.
Anat., 68, 457 (1938).

\section{Floral Meristem-organizing Gradient in Tobacco Stems}

THE existence of a gradient reflecting the ability to form flower buds, along the stems of tobacco plants (var. 'Wisconsin 38 '), has been previously reported ${ }^{1}$. Internode fragments, cultured on a simple medium devoid of growth substances, produce a callus which reflects this gradient in terms of its tendency to organize. Fragments isolated from the basal part of the stem produce only callus, those from the vegetative mid-part of the stem may produce 2 or 3 vegetative buds ${ }^{2}$, whereas those isolated from the flowering part of the stem form calluses producing 10 to 20 flowering buds ${ }^{3}$. The higher the fragments are cut, the greater the ratio of flower to vegetative buds per callus.

The gradient thus observed on internode fragments cultured in vitro probably exists in the intact flowering plant. The problem is to provoke a tissue proliferation in vivo, such that it bears newly formed organs, which would enable one to determine whether organ formation reflects such a gradient. The fact that tumours induced by certain strains of Agrobacterium tumefaciens can organize ${ }^{4-6}$ might prove useful in uncovering the existence of a gradient in vivo.

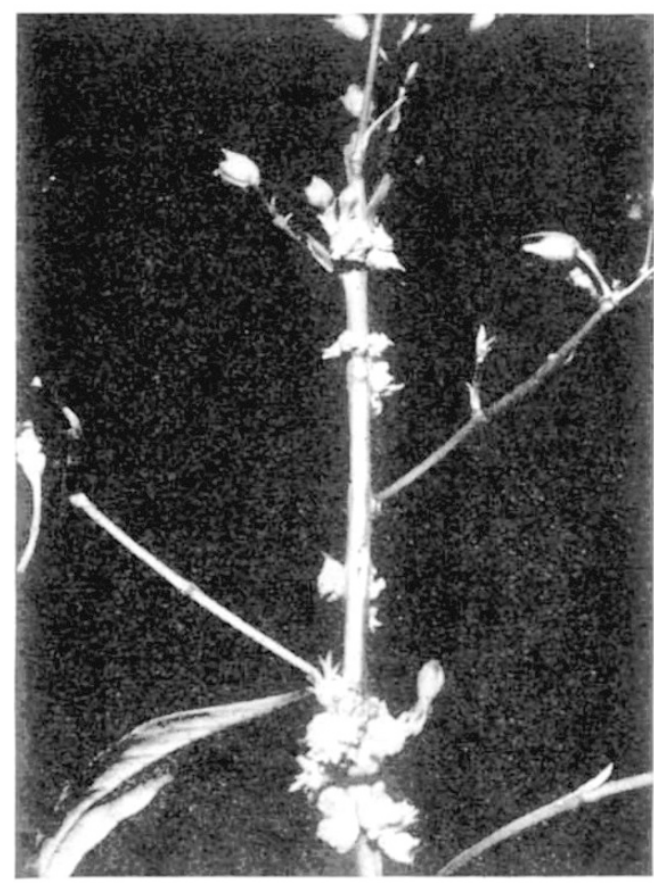

Fig. 1. Floral organogenesis on crown-gall tumour developed on Nicotiana tabacum, 'Wisconsin 38 '
Agrobacterium tumefaciens (Strain T37) was injected into the internodes of flowering Nicotiana tabacum plants (var. 'Wisconsin 38'). At the inoculation sites, tumours developed and organized, the nature of the organization depending on the level of the stem at which the injection was made. On the flowering part of the stem, the tumours were small, and bore organs some of which produced flower-buds (Fig. 1). Just underneath the inflorescence the tumours developed small stems with leaves and then flower-buds. Lower still, teratologic organoids were observed, and further towards the base of the stem only unorganized tumours were obtained. A flowering gradient was thus observed, and the gradient apparently exists in the intact plant as well as in isolated fragments.

Both in vivo and in vitro, the tendency for teratomatous tissue of tobacco to organize depends on the hormonal milieu in which such tissue is growing, particularly in respect to auxin and gibberellin ${ }^{6}$. For normal tobacco callus, the kinins also appear to play a critical part in bud organization? ${ }^{7}$ The gradient of organogenesis described in these preliminary experiments is therefore presumably due to a gradient of hormone concentrations inside the plant, but whether the flowering of the organs formed on the inflorescence-axis is entirely a hormonally controlled process cannot be decided until floral organogenesis can be experimentally induced with specific hormones. I thank Dr. Manigault for kindly supplying the bacterial strain.

\section{Danielle Aghion-Prat}

Laboratoire du Phytotron,

Gif-sur-Yvette, France.

${ }^{1}$ Chouard, P., and Aghion, D., C.R. Acad. Sci., Paris, 252, 3864 (1961).

${ }^{2}$ Skoog, F., and Tsui, C., Amer. J. Bot., 35, 782 (1948).

${ }^{3}$ Aghion, D., C.R. Acad. Sci., Paris, 255, 993 (1962).

4 Braun, A. C., Bot. Gaz., 114, 363 (1953).

${ }^{5}$ Braun, A. C., and Stonier, T., Protoplasmatologia, Band X, 5a, 1 (1958).

${ }^{6}$ Stonier, T., in Regeneration; 20th Growth Symp. Soc. Study of Development and Growth, 85 (1962).

Skoog, F., and Miller, C. O., in The Biological Action of Growth Substances, Symp. Soc. Exp. Biol. 11, 118 (1957).

\section{Influence of Ioxynil on the Photo-reduction of Endogenous Plastoquinone by Isolated Chloroplasts of Vicia faba $\mathbf{L}$.}

Although plastoquinone (a 2,3-dimethyl $p$-benzoquinone, with a $\mathrm{C}_{45}$ side chain at $\mathrm{C}_{5}$ ) was first isolated in 1946 by Kofler from alfalfa grass, it did not excite much interest until Cran ${ }^{1}$ extracted the compound from the chloroplasts of green leaves. Plastoquinone (PQ) was first implicated as a participant in photosynthetic electron transport systems by Bishop ${ }^{2}$. Recently, Redfearn et al. ${ }^{3}$ have shown plastoquinone to be an intermediary electron carrier in the photosynthetic system, being a part of the light-dependent water-splitting mechanism as outlined by Duysens et al. ${ }^{4}$. We have recently reported ${ }^{5}$ that ioxynil (4-hydroxy-3,5-diiodobenzonitrile) ${ }^{6}$ inhibits photophosphorylation and the accompanying reduction of pyridine nucleotide by chloroplasts. The results reported have been improved in recent months to give 50 per cent inhibition of both processes at a concentration of ioxynil of $5 \times 10^{-5} \mathrm{M}$. In an attempt to elucidate the action of ioxynil on the photosynthetic electron transport system, the ability of chloroplasts to photo-reduce endogenous plastoquinone was investigated.

Chloroplasts were isolated and washed as previously described ${ }^{5}$. The technique involving the measurement of plastoquinone was that described by Redfearn ${ }^{3}$ in which the steady-state oxidation-reduction levels are measured. Replicate tubes contained $0.05 \mathrm{M} \mathrm{Na} / \mathrm{K}$ phosphate buffer $p \mathrm{H} 7.0$ containing $0.01 \mathrm{M}$ potassium chloride, and chloroplasts equivalent to $1 \mathrm{mg}$ chlorophyll $/ \mathrm{ml}$. in a final volume of $3.0 \mathrm{ml}$. Other chemicals were added as required. The tubes were illuminated from both sides by two 